\title{
Using L dwarfs with distances to test models
}

\author{
Youfen Wang, ${ }^{1,2}$ H. R. A. Jones,${ }^{3}$ R. L. Smart, ${ }^{4}$ and Z. Shao ${ }^{1}$ \\ ${ }^{1}$ Shanghai Astronomical Observatory, 80 Nandan Road, Shanghai 200030, China \\ email: youfenwang@shao.ac.cn \\ ${ }^{2}$ Graduate University of the Chinese Academy of Sciences, 19 Yuquan Road, Beijing 100049, \\ China \\ ${ }^{3}$ University of Hertfordshire, Hatfield AL10 9AB, UK \\ ${ }^{4}$ Osservatorio Astrofisico di Torino, Strada Osservatorio 20, 10025 Pino Torinese, Italy
}

\begin{abstract}
We test two contemporary low-mass atmospheric models using three L dwarfs with distances and published spectra. We find that the two models do not predict the same trends for temperature, gravity and metallicity in absorption lines. We find that one model appears to better reflect the temperature, but this sample is too small to investigate the other parameters in depth.
\end{abstract}

Keywords. stars: low-mass, brown dwarfs, stars: distances

\section{Introduction}

L-type dwarfs are ultracool objects; they are cooler than M dwarfs. Most L dwarfs are expected to be brown dwarfs, i.e. they do not reach the hydrogen-burning phase. Parallax is a model-independent parameter which can be used to constrain stellar radius or temperature, and hence test and improve models. Considering that distances are so valuable, it is a sign of the difficulty in obtaining them that, of more than 800 known L dwarfs, fewer than 80 have measured parallaxes (Dupuy \& Liu 2012). In Wang et al. (2012) we published the parallaxes of five L dwarfs found using the robotic Liverpool Telescope (Steele et al. 2004). Using their measured astrometric parameters in combination with the stellar evolution model of Chabrier et al. (2000), we find that these objects are single thin disk-objects of solar metallicity, with gravities between $\log g=5.0$ and 5.5 (in cgs units) and temperatures as indicated in the first line of Table 1 (discussed below). Here we compare published optical spectral of three of these five objects to synthetic spectra from two widely used atmospheric models.

\section{The targets, models and spectra}

The three objects with published optical spectra (Cruz et al. 2007) are 2MASs J0141032 +180450 , 2MASs J1807159+501531 and 2MAss J2238074+435317 (hereafter 2M0141+ 1804, 2M1807+5015 and 2M2238+4353, respectively). Model spectra are from Burrows et al. (2006; hereafter BSH06) and Allard et al. (2009; http://phoenix.ens-lyon.fr/ Grids/Papers/, hereafter BT-DusTY). The BSH06 models cover $\log g=4.5,5.0$ and 5.5 and effective temperatures from 700 to $2000 \mathrm{~K}$, with metallicities of $[\mathrm{Fe} / \mathrm{H}]=-0.5,0,+0.5$ dex. The BT-DusTy models cover $\log g$ of 4.5, 5.0 and 5.5, and effective temperatures from 1500 to $3500 \mathrm{~K}$, with metallicities of $[\mathrm{Fe} / \mathrm{H}]=-0.5,0,+0.5$. In addition to the temperatures derived from a comparison to Chabrier et al. (2000), in Table 1 we list the temperatures obtained from an application of the Stefan-Boltzman law, where the bolometric luminosity was estimated from a combination of the observed and model spectra. 
Table 1. Derived temperatures

\begin{tabular}{cccc}
\hline Obj. name & $2 \mathrm{M} 0141+1804$ & $2 \mathrm{M} 1807+5015$ & $2 \mathrm{M} 2242+2542$ \\
\hline$T_{\text {eff }, \mathrm{m}}(\mathrm{K})$ & $2225-2305$ & $2000-2138$ & $1688-1850$ \\
$T_{\text {eff }, 1}(\mathrm{~K})$ & $2147-2216$ & $1999-2085$ & $1736-1844$ \\
$T_{\text {eff }, 2}(\mathrm{~K})$ & $2126-2206$ & $1982-2071$ & $1699-1823$ \\
\hline
\end{tabular}

Notes: $T_{\text {eff }, \mathrm{m}}$ indicates the value derived from the Chabrier et al. (2000) evolutionary model and our parallaxes (Wang et al. 2012); $T_{\mathrm{eff}, 1}$ and $T_{\mathrm{eff}, 2}$ are calculated from the Stefan-Boltzman law, where the bolometric luminosity was estimated from a combination of the observed and the BT-DUSTY and BSH06 model spectra, respectively.

The three values in the temperature range for each individual target are consistent, except for the relatively large discrepancy between $T_{\text {eff }, 1}$ and $T_{\text {eff }, 2}$ for target $2 \mathrm{M} 2242+2542$. This could indicate a problem with these models for the lower-temperature objects.

\section{Comparisons of L1 models}

$\mathrm{L}$ dwarfs have absorption lines that are different from $\mathrm{M}$ dwarfs: see Kirkpatrick et al. (1999), i.e. the paper which defines the L spectral type. Instead of the prominent TiO and VO lines, metallic hydrides and neutral alkali lines are the major characteristics in the far optical band, especially for mid-late L dwarfs (Kirkpatrick 2005). Below we test how the temperature, gravity and metallicity affect these characteristic lines and compare the models with the observational spectra described above.

In Fig. 1 we compare wavelength versus flux for the BSH06 and BT-DUSTY model spectra. In each panel we plot the flux of one spectrum divided by the other to test which has stronger absorption lines: if the divided flux yields lines in absorption, then the numerator spectrum has stronger absorption lines. In Fig. 1, panels (a) and (b), we test temperature differences. The BSH06 models yield very obvious KI and RbI absorption lines, which indicates that the cooler object has stronger KI and RbI absorption lines. However, the BT-DusTy spectra do not follow the same trend; these models yield much weaker KI and RbI lines. In Fig. 1, panels (c) and (d), we test different gravities. The alkali-line changes of the two models are consistent, except for the KI lines. The BT-DusTY models yield weaker absorption lines in panel (d), indicating that the BT-DUSTY model is not as sensitive to gravity as BSH06. In panels (e) and (f) we test different metallicities. The BSH06 spectra predict that the metal-poor objects have stronger absorption lines, while the BT-DusTy spectra predict that metal-rich objects have stronger absorption lines. This analysis has not considered NaI and other alkali lines, since corresponding changes of these lines are not prominent, which might be a problem for both models.

In Fig. 2 we plot the observational optical wavelength-flux diagram of the three targets, $2 \mathrm{M} 0141+1804,2 \mathrm{M} 1807+5015$ and $2 \mathrm{M} 2238+4353$, as well as a standard L1: $2 \mathrm{MASSW}$ J1439284+192915 (labelled 2M1439+19; from Kirkpatrick et al. 1999) which has an effective temperature range of 2100-2200 K, and a standard L3: DENIS-P J1058.7-1548 (labelled Denis-P J1508-15; from Delfosse et al. 1997), which has an effective temperature of 1700-1950 K (Leggett et al. 2002). When we divide our observed spectra by one another-(a) L3/L1, (b) L1.5/L1, (c) L1.5/L1 and (d) L1.5/L1.5 - we always keep the cooler spectrum as the numerator. We also indicate the temperature difference in each plot. The significant difference of molecular bands for (a),(b), (c) and (d) is caused by the disappearance of $\mathrm{TiO}$ and VO absorption bands in mid-late $\mathrm{L}$ dwarfs. From Fig. 2 we see that the cooler objects have stronger alkali and metallic-hydride absorption lines. Panels (a) to $(\mathrm{d})$ form a sequence of decreasing $\Delta T$ and, thus, decreasing 


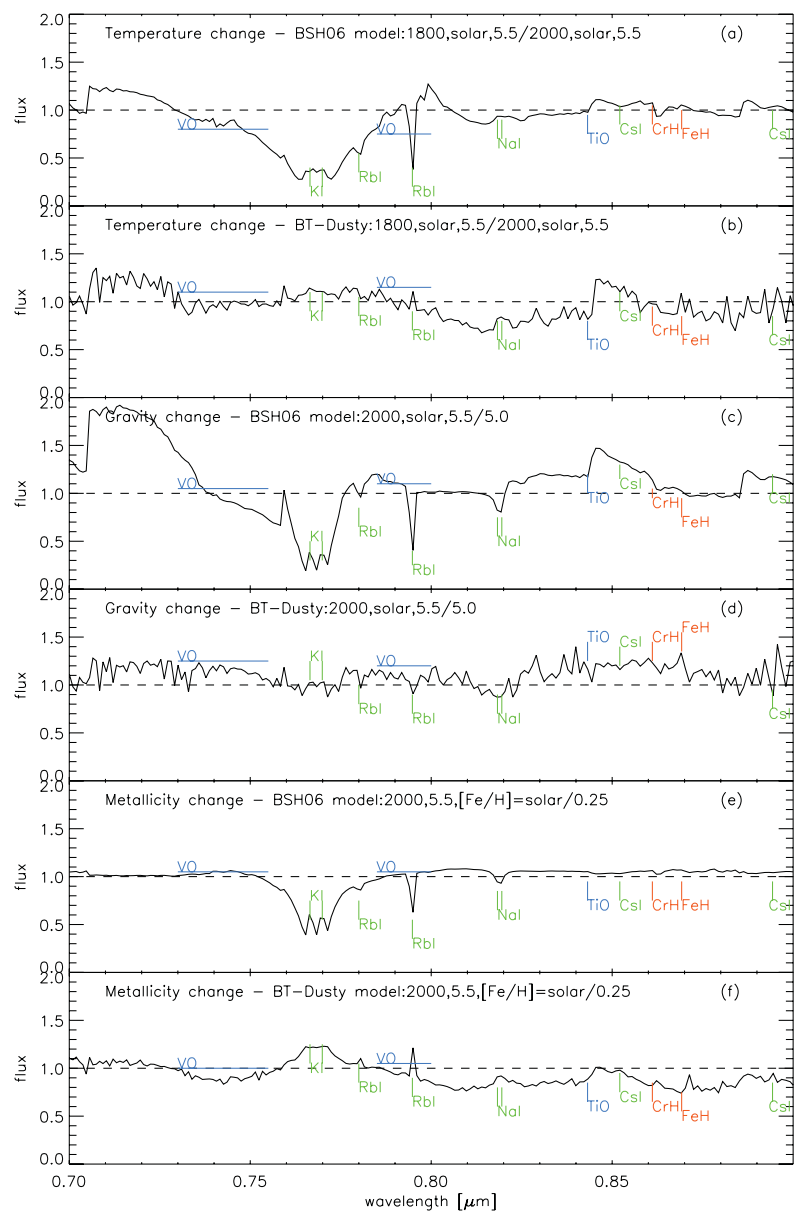

Figure 1. Wavelength-flux diagrams of synthetic optical spectra divided by one another to highlight differences between them. Model spectra are normalised before we do the division. We rebinned high-resolution BT-DUSTY model spectra to match the lower-resolution BSH06 models. Panels (a), (c) and (e) use BSH06, whereas panels (b), (d) and (f) are based on BT-Dusty. Comparisons (a) and (b) are for a temperature change of $200 \mathrm{~K}$, (c) and (d) are for a gravity change of 0.5 , and (e) and (f) are for a metallicity change of 0.25 dex.

absorption-line strength. Nonetheless, it is notable that the change in atomic absorption features in plots (b) and (c) is not dissimilar to that in (a), suggesting that there may be changes in absorption-line strength (at similar spectral type) caused by metallicity or gravity.

We now return to the two models, BHS06 and BT-DusTy, especially, considering the different predictions for the changes in the absorption lines they made for changes in temperature. Fig. 2 is more consistent with the predictions of the BSH06 models. We will continue to make a more robust comparison in gravity and metallicity parameter space when we have a larger sample and larger variation in these parameters.

\section{Acknowledgements}

This work is partially funded by IPERCOOL no. 247593 International Research Staff Exchange Scheme and PARSEC no. 236735 International Incoming Fellowship within the Marie Curie 7th European Community Framework Programme. RS and HRAJ 


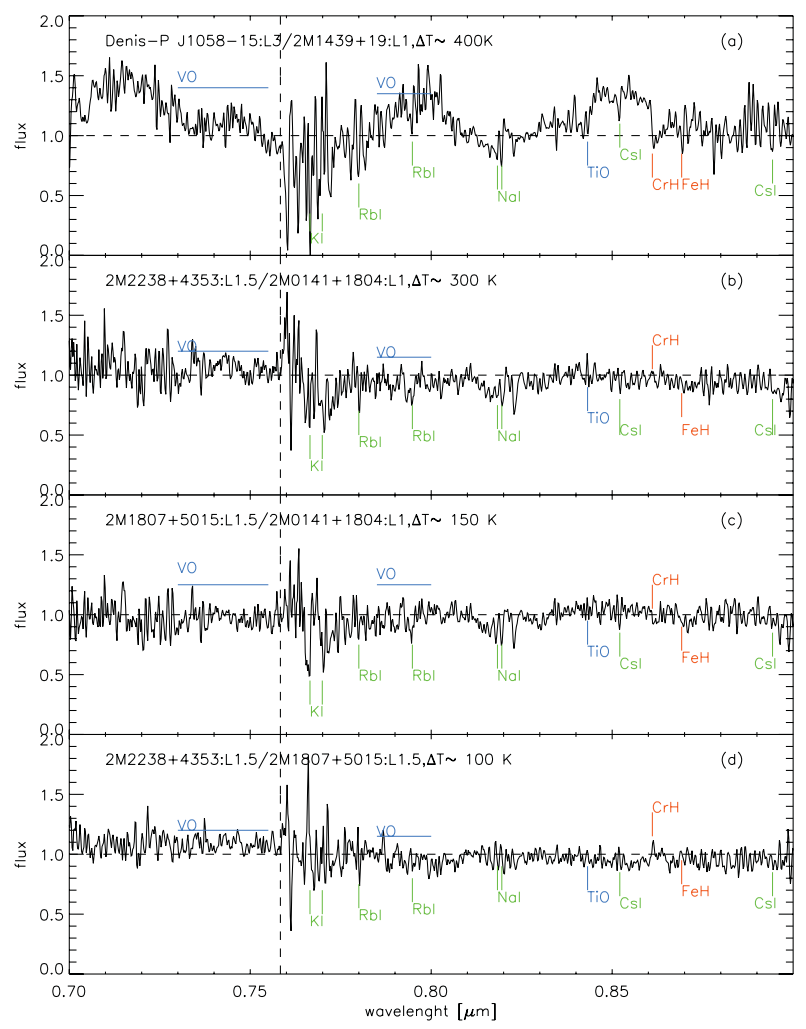

Figure 2. Wavelength-flux diagrams of observational optical spectra divided by spectra of later types. The target spectra are supplemented by one standard L3 and one standard L1. They are rebinned and normalised before the division. The cooler spectrum is always divided by the hotter one. Panels (a) to (d) form a decreasing sequence of $\Delta T$. The vertical dashed line indicates the position of telluric lines near the potassium line.

acknowledge the support of Royal Society International Joint Project 2007/R3. The Liverpool Telescope is operated on the island of La Palma by Liverpool John Moores University in the Spanish Observatorio del Roque de los Muchachos of the Instituto de Astrofísica de Canarias with financial support from the UK Science and Technology Facilities Council. We thank K. L. Cruz for kindly providing the spectra.

\section{References}

Burrows, A., Sudarsky, D., \& Hubeny, I. 2006, ApJ, 640, 1063

Chabrier, G., Baraffe, I., Allard, F., \& Hauschildt, P. 2000, ApJ, 542, 464

Cruz, K. L., Reid, I. N., Kirkpatrick, J. D., et al. 2007, AJ, 133, 439

Delfosse, X., Tinney, C. G., Forveille, T., et al. 1997, A\&A, 327, L25

Dupuy, T. J. \& Liu, M. C. 2012, ApJ, 201, 19

Kirkpatrick, J. D., Reid, I. E., Liebert, J., et al. 1999, ApJ, 519, 802

Kirkpatrick, J. D. 2005, ARA\&A, 43, 195

Smart, R. L., Lattanzi, M. G., Bucciarelli, B., et al. 2003, A\& A, 404, 317

Steele, I., Smith, R. J., Rees, P. C., et al. 2004, Proc. SPIE, 5489, 679

Wang, Y., Jones, H. R. A., Smart, R. L., et al. 2012, MNRAS, submitted 